\title{
Tissue regeneration using endothelial colony-forming cells: promising cells for vascular repair
}

\author{
Kimihiko Banno ${ }^{1}$ and Mervin C. Yoder ${ }^{1}$
}

Repairing and rebuilding damaged tissue in diseased human subjects remains a daunting challenge for clinical medicine. Proper vascular formation that serves to deliver blood-borne nutrients and adequate levels of oxygen and to remove wastes is critical for successful tissue regeneration. Endothelial colony-forming cells (ECFC) represent a promising cell source for revascularization of damaged tissue. ECFCs are identified by displaying a hierarchy of clonal proliferative potential and by pronounced postnatal vascularization ability in vivo. In this review, we provide a brief overview of human ECFC isolation and characterization, a survey of a number of animal models of human disease in which ECFCs have been shown to have prominent roles in tissue repair, and a summary of current challenges that must be overcome before moving ECFC into human subjects as a cell therapy.

$\mathbf{M}$ ethods to treat pediatric patients who suffer from tissue and organ damage acquired prenatally or postnatally remain elusive and largely experimental for pediatric clinicians and surgeons. The causes of tissue and organ damage are multifaceted and include congenital anomalies that predispose to ongoing tissue injury, inadequate or excessive vascularization, hypo-perfusion, hypo- or hyperoxygenation, inflammation, or trauma. In the neonatal period, hypoxic-ischemic encephalopathy, bronchopulmonary dysplasia (BPD), retinopathy of prematurity, or necrotizing enterocolitis are some representative disorders that are well known to cause severe tissue damage as a consequence of perinatal asphyxia or are associated with complications of premature birth. These disorders are currently treated conservatively in hopes of spontaneous tissue recovery or in some instances repair is promoted by surgically removing the most damaged and non-functional tissue. Although overall outcomes continue to improve for these illnesses (1-5), many infants develop chronic illness due to consequences of the acute injuries to these organ systems.

One system that is critical for the long-term repair of the above illnesses is the vascular system. Tissue-regenerative approaches using endothelial progenitor cells (EPCs) would be an attractive approach for repairing or regenerating vasculature in all of these illnesses (6,7), if current basic investigation could be translated into human subjects. Endothelial colony-forming cells (ECFCs) are the most potent vascular reparative cell type among EPC candidates (7). In this review, we describe the identifying features of ECFCs and define the sources of ECFC that include tissue-resident vascular endothelium, human umbilical cord, or peripheral blood, and are also from human-induced pluripotent stem cells (hiPSCs). We also provide an overview of preclinical studies where human ECFCs have been administered into animal models of human disease, focusing on those studies that will be feasible for pediatric clinical application. Finally, we provide an update on current limitations for translating the exciting potential of human ECFC in the preclinical studies into human clinical trials.

\section{ECFC AS A PROMISING CELL SOURCE FOR TISSUE REGENERATION}

Blood vessels may have a direct or indirect role in the process of tissue injury. Regardless of the pathophysiology, breakdown of the blood vessel wall with loss of endothelial barrier properties is an acute emergency that requires prompt platelet, inflammatory cell, and pro-coagulant protein activation and aggregation (clot formation) to stop the escape of intravascular blood cells and bleeding into the tissue $(8,9)$. Restitution of blood flow to the injured site may require angiogenic sprouting of endothelial cells from nearby intact blood vessels to generate new blood vessels as well as vasculogenesis by circulating ECFC to recanalize the clot to permit new blood vessel invasion to restore tissue perfusion. We provide an overview of this process and highlight some of the cells that may have important roles in Figure $\mathbf{1}$.

We first consider the most current definition of human EPCs. Putative human EPCs have been identified using many approaches (8). Myeloid angiogenic cells (also called circulating angiogenic cells (CACs), pro-angiogenic hematopoietic cells (PAC), pro-angiogenic circulating hematopoietic stem/ progenitor cells, or "early" EPCs; Figure 2), have all been defined as EPCs (9-11) and they have important roles in the process of vessel injury and repair (12). However, these MAC, 


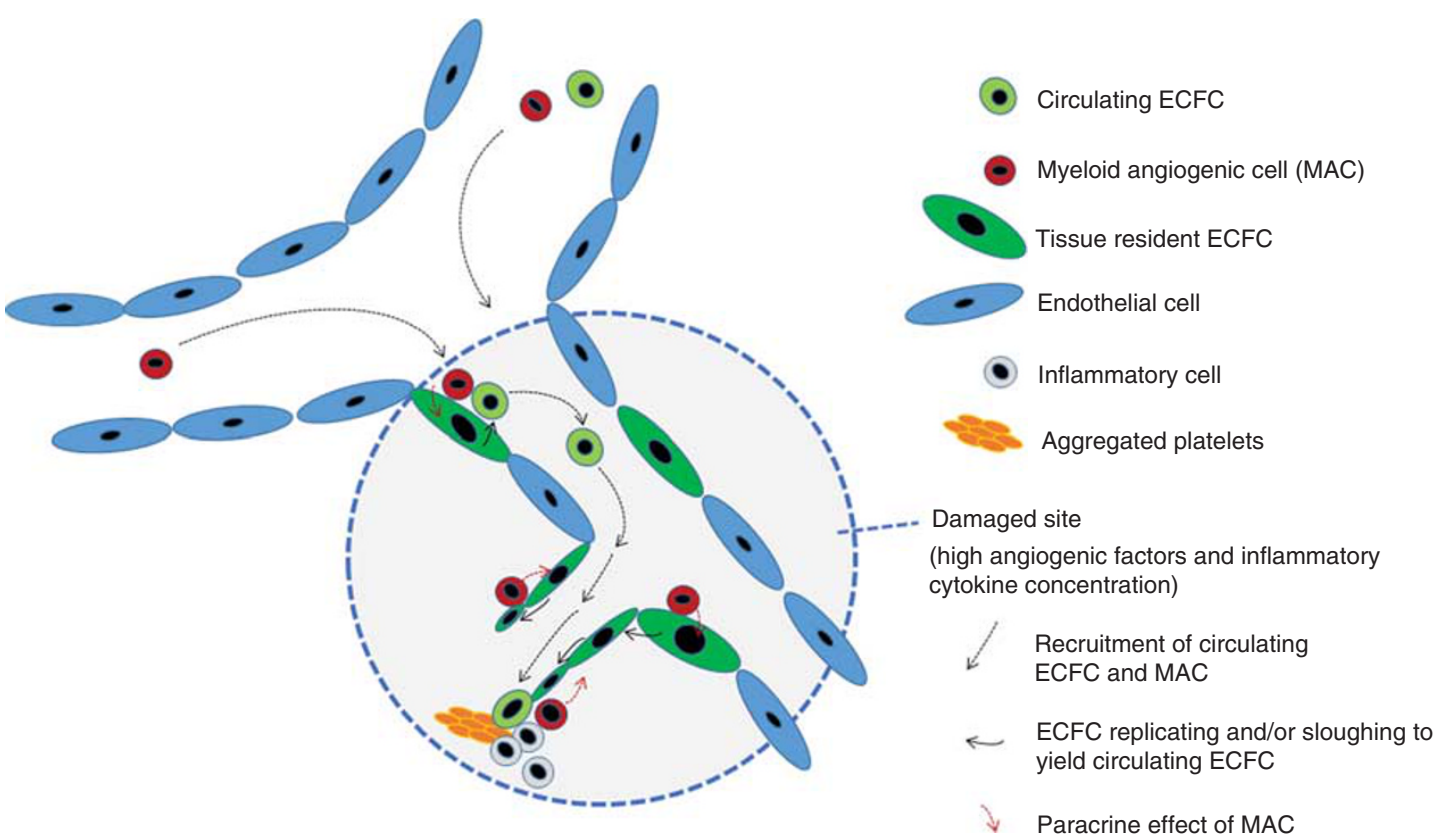

Figure 1. Circulating myeloid angiogenic cells (MACs) are delivered into the damaged tissue where angiogenic factors and inflammatory cytokine concentration are higher than other tissues. Paracrine factors of MACs recruit endothelial colony-forming cells (ECFCs) from either the circulation or the local vascular wall. Next, migration and proliferation of ECFCs, which are guided by MACs, restore the endothelial integrity of the vascular wall. Circulating ECFCs may arise form tissue-resident ECFCs and are recruited to locally damaged sites, and contribute to re-vascularization.

CAC, PAC, pro-CPC, and "early" EPCs do not represent actual endothelial progenitors in that none of these cells ever change their lineage fate and fully integrate as bona fide endothelial cells into the remodeled endothelium $(13,14)$; rather, MAC, CAC, PAC, pro-CPC, and "early" EPC represent hematopoietic cells that support endothelial repair and regeneration in injured vessels through largely paracrine mechanisms (7). In contrast, ECFCs (also called outgrowth endothelial cells or "late" EPCs; Figure 2) represent an endothelial cell type with potent intrinsic clonal proliferative potential and capacity to contribute to de novo blood vessel formation in vivo $(6,15)$. Although ECFCs and MACs are intrinsically different lineages of cells (16), they cooperate in the re-vascularization process (Figure 1). First, circulating MACs are delivered into the damaged tissue and their paracrine factors recruit ECFCs from either the circulation or the local vascular wall (12). Next, migration and proliferation of ECFCs, which are guided by MACs, are recruited to the injured site to restore the endothelial integrity of the vascular wall $(17,18)$. Although the exact origin of circulating ECFC remains unclear, some authors have proposed that circulating ECFCs arise from tissue-resident ECFCs, are recruited to locally damaged sites, and contribute to re-vascularization (19). Taken together, based on current accumulating evidence, ECFCs are the most rationale and promising cell source that are able to directly incorporate into or directly form (postnatal vasculogenesis) regenerating vessels in areas of tissue regeneration.

\section{ECFC DERIVED FROM UMBILICAL CORD BLOOD OR PERIPHERAL BLOOD}

Ingram et al. identified a novel hierarchy of ECFCs based on their clonogenic and proliferative potential, analogous to the hematopoietic cell system (20). After they harvested mononuclear cells isolated from adult peripheral and umbilical cord blood (CB) and cultured the cells on type I rat tail collagencoated dishes as adherent cells, proliferating colonies of ECFC were identified by their cobblestone appearance and lack of hematopoietic phenotype and functions. Some but not all of these colonies showed high proliferative potential (HPPECFCs). defined as the ability to clonally expand in vitro into more than 2,000 endothelial cells in 14 days and to be capable of replating into secondary HPP-ECFC in which some clones display the same capacity to form colonies of $>2,000$ cells in 14 days (18). These ECFCs not only possess robust proliferative potential but also have capacity to form new blood vessels in vitro and in vivo (17,20-24). Although human ECFCs have not been shown to express any novel antigens that permit them to be differentiated from non-proliferative vascular endothelial cells, they can be identified by expression of the cell surface proteins CD31, CD34, Kinase Insert Domain Receptor (KDR), CD144, CD105, CD146, and von Willebrand factor, and they do not express the proteins CD45, CD14, CD115, and AC133 that are indicative of hematopoietic cells (21) (Figure 2).

\section{hiPSC-DERIVED ECFCs}

hiPSCs have been readily established by reprogramming a host of somatic cell types using defined transcription factors 


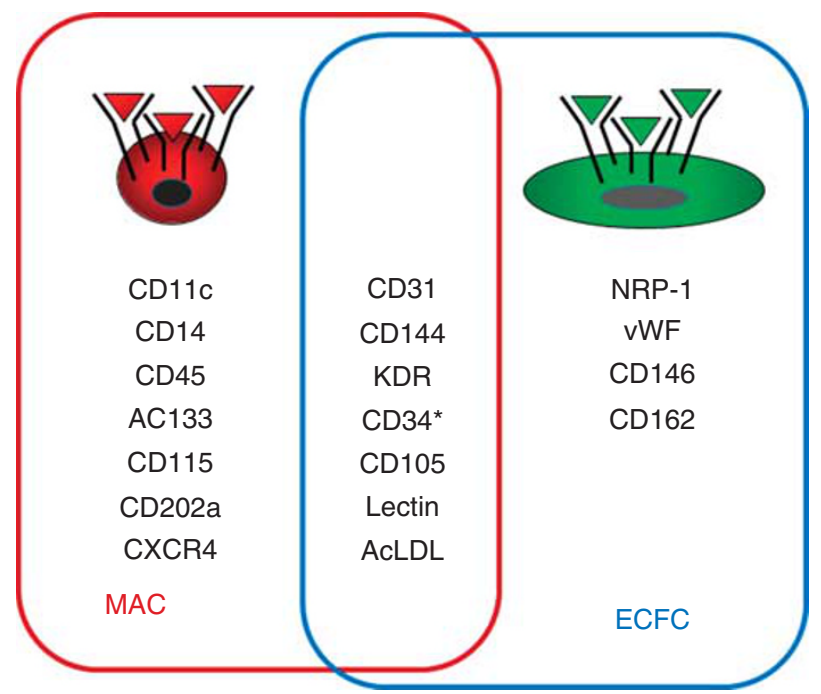

Figure 2. Cell surface markers that identify myeloid angiogenic cells (MAC; left, red circle; also called circulating angiogenic cells (CACS), proangiogenic hematopoietic cells (PAC), pro-angiogenic circulating hematopoietic stem/progenitor cells (pro-CHSPCs or pro-CPCs), or "early" endothelial progenitor cells (EPCS)) and endothelial colony-forming cell (ECFC; right, blue circle; also called outgrowth endothelial cells (OECs), or "late" EPCs). The common markers for both MAC and ECFC are located in center circle. Although CD34, KDR, and AC133 are the most useful markers for MAC, a host of other markers have also been shown to identify hematopoietic cells that assist in vascular repair and regeneration via the paracrine secretion of growth factors, chemokines, matrix metalloproteinases, but do not change their lineage fate to become endothelial cells that form new blood vessels in vitro or in vivo. Thus, MAC are not true "endothelial progenitor cells" as originally reported (9). Note that MAC can be isolated from the $\mathrm{CD} 34^{+}$population; however, the level of CD34 expression will change in culture (shown in asterisk). CD31, CD34, CD144, and KDR are the most useful markers for ECFC, whereas CD14 and CD45 are not expressed at the protein or mRNA level in these cells (19). A host of other markers have also been shown to identify this endothelial cell type that clonally proliferate and contribute to de novo blood vessel formation in vivo (see (ref. 19) for detailed summary).

or small molecules (25). The hiPSCs that emerge following cell reprogramming possess unlimited self-renewal capacity and display the ability to differentiate into any cell population. Although many successful attempts have been conducted to generate endothelial cells from PSCs, the derived cells typically display low proliferative potential and often undergo an endothelial-to-mesenchymal transition within five to eight passages (26-29). Prasain et al. successfully differentiated hiPSCs into cells that display clonal proliferative potential, in vivo vessel-forming ability, and gene expression that is similar to CB-ECFCs (30). This protocol focused on isolating $\mathrm{CD} 31^{+}$Neuropilin- $1^{+}$cells $\sim 12$ days into the hiPSC differentiation process to identify the cells that give rise to a homogenous population of ECFC. The proliferation and expansion of the hiPSC-derived ECFC depends upon sustained activation of KDR signaling (augmented by Neuropilin-1) through addition of vascular endothelial growth factor A $\left(\mathrm{VEGF}_{165}\right)$. The derived ECFCs not only showed a stable endothelial phenotype with high clonal proliferative potential and the capacity to form functional human blood vessels in immunodeficient mice (for up to 6 months post implantation), but also formed new blood vessels in the damaged retina and ischemic hindlimb of experimental mouse models of human disease (28). These results suggest that hiPSC-ECFC may serve as a robust source of ECFC for human clinical trials.

\section{PRECLINICAL STUDIES USING ECFCS WITH RELEVANCE TO PEDIATRIC DISEASES}

Although unilateral hind limb ischemia or myocardial infarction animal models of adult human cardiovascular disease are frequently used to examine human ECFC treatments $(31,32)$, we have decided to focus in this review on the preclinical studies in which ECFCs were used as a therapeutic method that will have relevance for pediatric diseases.

\section{BRAIN}

Traumatic brain injury is a significant cause of morbidity and mortality in children (33). Zhang et al. first performed transplantation of human CB-ECFCs intravenously to a mouse model of traumatic brain injury and revealed that donor ECFCs were detected in injured brain and increased the microvessel density resulting in significantly less neurologic disability (34). The same group administered CB-ECFCs into the ventricles of the injured brain and the infused ECFCrestored blood-brain barrier integrity and promoted angiogenesis (35). In an ischemic stroke mouse model, green fluorescent protein-labeled CB-ECFCs administered via intraarterial injection were found surrounding the infarct border zone in brain sections and contributed to functional restoration, improved angiogenesis, neurogenesis, and overall decreased apoptosis (36). Intravenously transplanted CBECFC in a rat model of transient middle cerebral artery occlusion also homed to the ischemic hemisphere and improved functional recovery (37). Recently, erythropoietin pretreatment of transplanted ECFCs enhanced recovery in the rat middle cerebral artery occlusion model. Cerebral blood flow was significantly restored with ECFC treatment, but erythropoietin-priming further enhanced functional recovery through an increased homing of ECFCs to the ischemic hemisphere (38). Taken together, therapeutic usage of ECFCs is a promising strategy to enhance brain tissue viability and function in cerebral traumatic or ischemic injury in several animal models of human disease. Further investigation of the effects of infused ECFC in other animal models more reflective of human neonatal hypoxia and ischemia are needed.

\section{LUNG}

Before attempting therapeutic usage of ECFCs in infant lung injury, the investigators first focused on intrinsic characteristics of circulating ECFCs in several disease conditions. Changes in the number and/or dysfunction of CB-ECFCs were examined and reported in infants or children that later 


\section{Review $\mid$ Banno and Yoder}

developed specific lung disorders, such as BPD. Preterm $\mathrm{CB}$ yielded significantly more ECFC colonies than term $\mathrm{CB}$, and preterm ECFCs demonstrated increased growth but enhanced apoptotic susceptibility to hyperoxia (39). This detrimental effect may be related to disruption of VEGF-nitric oxide signaling (40). Interestingly, the number of CB-ECFCs in preterm babies who subsequently developed moderate and severe BPD were significantly decreased $(41,42)$, and lung ECFC number and function were impaired in experimental hyperoxia-induced BPD in newborn rats (43). Because impaired vascular growth has a central role in the pathogenesis of BPD, decreased ECFCs may contribute to abnormal vascular repair and regeneration in ventilated and oxygenexposed preterm infants resulting in BPD. To address this question, Alphonse et al. administered CB-ECFCs into a postnatal rat pup model of BPD and a single intravenous dose of ECFCs reversed the alveolar growth arrest, preserved lung vascularity, and attenuated pulmonary hypertension, both in short-term (1 month) and long-term (10 months) assessments (43). Recently, the isolation and culture of pulmonary microvascular ECFCs from human and rat lung tissue has become feasible within 4 weeks (44). This method will provide more accurate information about ECFCs in human lung disease and further contribute to the development of preclinical research in this field. Of interest, conditioned medium derived from cultured CB-ECFC has also been shown to prevent pulmonary hypertension in newborn pups exposed to bleomycin (45). These results suggest that some CB-ECFC-derived soluble molecules or membrane particles may deliver regenerative molecules to the injured rodent lung to enhance recovery from otherwise BPD-causing reparative responses.

\section{RETINA}

The mouse model of oxygen-induced retinopathy has been widely used in studies related to retinopathy of prematurity and/or proliferative diabetic retinopathy, and provided a useful tool for assessing angiogenesis in the postnatal mouse retina (46). When mouse pups exposed to hyperoxia were given intravitreally injected ECFCs, the human ECFCs directly incorporated into the damaged resident vasculature, significantly decreased the avascular areas caused by the hyperoxia, concomitantly increased normovascular areas, and prevented pathologic pre-retinal neovascularization (47). Significant reduction of avascular areas and pre-retinal neovacular tufts was similarly observed when hiPSC-derived ECFCs were injected into the vitreous of mouse pups exposed to hyperoxia (30). To achieve better treatment effects, Sakimoto et al. chose a subset of ECFCs with high CD44 expression levels. The injected CD $44^{\text {high }}$ ECFCs facilitated regeneration of retinal vasculature and reduced pathological angiogenesis compared with CD $44^{\text {low }}$ ECFCs (48). These studies provide evidence that human ECFCs may have an important role in rescuing the developing murine retina from the effects of high levels of oxygen and assist in the prevention of retinopathy of prematurity. Further studies to assess the mechanisms of ECFC protection, the impact of the revascularization on retinal function, and the persistence of the repair would be important for future work in this area.

\section{KIDNEY}

Acute kidney injury (AKI), formerly called acute renal failure, is commonly defined as an abrupt decline in renal function, and is mainly caused by ischemia/reperfusion injury (49). As ischemia/reperfusion induces significant vascular injury leading to capillary loss, the administration of exogenous endothelial cells has been studied as a potential strategy to treat AKI. Infusion of human umbilical vein endothelial cells into rats with AKI was first conducted by Brodsky et al. and a significant protection of the rodent kidneys from experimental AKI was reported (50). Administration of ECFCs also significantly attenuated increases in plasma creatinine, tubular necrosis, macrophage infiltration, oxidative stress, and apoptosis in the rodent AKI model (51). Similar effects were recently reported when rodent HPP-ECFC or human CBECFC were intravenously injected into rodents with AKI; furthermore, the preventative functions were present in paracrine factors secreted by the rodent and human ECFC (52). Other reports agree that the beneficial effects of ECFCs in preventing AKI damage to kidneys may not require cell engraftment (53), but do require ECFC-derived exosomes (51). Vinas et al. specifically identified microRNA in ECFCderived extracellular vesicles and reported that miR-486-5p enrichment in ECFC exosomes has a critical role in protecting kidney ischemia/reperfusion injury through targeting of the phosphatase and tensin homolog protein and the Akt pathway (54). This result suggests that the use of exosomes from ECFCs may be another strategy to protect against endothelial injury in animal models of AKI.

\section{CANCER}

During cancer progression, angiogenesis is always activated to sufficiently vascularize the tumor for uncontrolled growth, causing normally quiescent vasculature to continually sprout new vessels (55). To determine which vascular cell population specifically participates in this neoangiogenesis within the tumor tissue is critical not only for understanding the physiology of proliferating tumors but also for designing anti-angiogenic drug therapy. Naito et al. found that a small population of vascular-resident endothelial cells in lung predominantly contributed to a new blood vessel formation in mouse lung tumor models (56). This population was identified using the Hoechst side population method (57), and the cells isolated were shown to possess colony-forming ability in vitro, suggesting that these mouse tissue-resident vascular endothelial cells displayed characteristics similar to human ECFCs. Recently, human ECFCs have been generated as a targeting cell population for the tumor microvasculature carrying antitumor reagents as a novel cancer therapy (5860). For example, Laurenzana et al. engineered CB-ECFCs with a lentivirus encoding matrix metalloproteinase 12 (MMP12) to deliver the MMP12 as an antitumor urokinase- 


\section{Tissue regeneration using ECFCs $\quad$ Review}

type plasminogen activator (uPA) receptor-degrading enzyme, and showed that infusion of ECFCs generated significantly increased amounts of cleaved uPA receptor within the tumor cells and strongly inhibited tumor growth, tumor angiogenesis, and development of lung metastasis (59). Another interesting therapeutic approach utilized ECFCs enriched with chitosan-coated gold nanoparticles, which produced local hyperthermia when exposed to near-infrared light and generated sufficient heat to warm up the tumor environment to kill the cancer cells in vitro and in vivo (60). These data suggest that ECFCs may be usable of as a potential antiproliferative treatment for tumor tissue under certain circumstances.

\section{SUPPORTIVE CELLS AND REAGENTS}

Much effort has been devoted to determining which cells or reagents can enhance the neoangiogenic potential of ECFCs. A combination treatment of ECFCs with mesenchymal stem/ progenitor cells (61-65), adipose tissue-derived stromal cells (66), or host myeloid cells $(61,67)$ has been reported to synergistically enhance neovascularization compared with use of either cell population alone. Although some of these beneficial effects were implicated with the known immunosuppressive potency of mesenchymal stem cells (64) or priming ECFCs by Notch signaling (65), the exact underlying mechanism of interaction among these cells that promotes neovascularization still needs further investigation. In addition, pretreatment of ECFC with erythropoietin $(38,68)$, fucoidan $(69,70)$, the formyl peptide receptor-2 agonist WKYMVm (71), soluble CD146 (ref. 72), transmembrane tumor necrosis factor-a (73), and platelet lysate (74) are all candidate reagents that improve the therapeutic in vivo efficacy of administered ECFCs. Scaffold materials also have critical roles in vasculogenesis, and ECFCs can be incorporated within the scaffolds for generating a pre-vascularized tissue-engineered construct. Allen et al. compared rat tail type I collagen, bovine fibrin, and a synthetic peptide (PuraMatrix) as scaffolds into which ECFCs and mesenchymal progenitor cells were suspended and then implanted to form blood vessel networks in murine hosts. The suspended ECFCs and mesenchymal progenitor cells in any of the three matrices initiated a rapid onset of vascularization (75). Interestingly, Critser et al. reported that modulating the biomechanical properties of scaffolds via altering collagen fibril density and matrix stiffness were found to augment collagen implant remodeling as well as ECFC-derived vessel density, the proportion of human ECFC to host murine vessels, and total ECFC-comprised blood vascular area (76). Although a comprehensive survey in this field is beyond this review, further advances in application of scaffold materials that enable ECFCs to facilitate angiogenesis properly in each damaged tissue will surely advance the field of tissue regeneration.

\section{SUMMARY AND PERSPECTIVES (INCLUDING CURRENT LIMITATIONS)}

The above overview of results of some preclinical studies using human ECFCs provides evidence that ECFCs may represent a promising cell source for revasculogenesis in damaged tissue of pediatric patients. ECFCs possess outstanding vessel-forming potential in vivo, and their potential can be further augmented by addition of adventitial cells, biological reagents, and scaffolds. Before proceeding to the next step for clinical application, several practical issues need to be addressed.

First, we have not yet obtained a distinguishing marker for the HPP-ECFC that permits prospective isolation of these cells from peripheral blood or CB. Although the clonal assay of ECFC production can enumerate the number of HPPECFC in a given endothelial cell population, the HPP-ECFCinitiating cells never replicate via symmetric division and, thus, give rise to the entire ECFC hierarchy including low proliferative potential-ECFC, ECFC clusters, and nonproliferating endothelial cells within the HPP-ECFC colonies that emerge (18). Thus, the research field continues to search for cell surface markers that may prospectively isolate and enrich for HPP-ECFC. Second, some of the current reagents for ECFC isolation are derived from animal sources (such as type I rat tail collagen or fetal bovine serum). However, there is a new xeno-free media preparation EC-Cult-XF ECFC (Stemcell Technologies, Vancouver, BC, Canada), which might help to solve this issue after approval for clinical use. Third, use of autologous blood for ECFC isolation is currently not feasible in pediatric patients. ECFCs circulate in the blood stream at 1 per $10^{6}-10^{8}$ peripheral mononuclear cells (18). They may be isolated in sufficient numbers from umbilical $\mathrm{CB}$ for potential use in newborn infants; however, once processed and frozen, the CB-ECFC would need to be banked and fees for this kind of service can be prohibitive. In addition, the rationale for banking a cell product that is specified to a single lineage (endothelium) as a source of reparative cells that may not be needed until late adulthood is questionable at this point. For older children and adult subjects, primary circulating ECFCs from peripheral blood (PB-ECFC) are less clonogenic, proliferative, and angiogenic than CB-ECFCs. Finally, it is well known that certain disease states, such as diabetes, can significantly diminish the frequency and function of the isolated ECFC to such an extent that these cells would not be of sufficient quality for use as a revascularization therapy $(77,78)$.

One strategy to overcome the limited availability of autologous ECFC would be through the generation of patient-specific hiPSC-derived ECFCs (30). Our current protocol enables us to convert hiPSCs into cells similar to CB-ECFCs at an efficiency of $>10^{8}$ ECFCs produced from each starting iPSC within 80 days. However, this approach requires some (months) time for derivation of the autologous hiPSCs from each patient, in addition to differentiation of the patient-specific hiPSCs to ECFCs. Haplotype-based banking 
of hiPSCs might be an alternative approach, which can reduce the host immunogenic responses to the hiPSC-derived cells $(79,80)$. In this approach, individuals with homozygous human leukocyte antigen (HLA) haplotypes could be chosen for production of iPS cell lines, and the establishment of an HLA-organized bank would be sufficient for a large number of recipients. By creating a bank of the HLA haplotypes, one could readily match the patient with the appropriate HLA haplotype and then differentiate the cells to ECFC for injection into the patient. Another interesting approach to reduce allograft rejection of transplanted ECFCs utilizes clustered regularly interspaced short palindromic repeat (CRISPR)/Cas9-modified ECFC lacking class II major histocompatibility complex molecules (81) and the implanted synthetic microvessels, formed from the manipulated cells, were significantly protected from $\mathrm{CD}^{+}$T-cell-mediated destruction in vivo. However, implanting a cell source that lacks appropriate major histocompatibility complex class II markers that permits escape from immune detection may be a risk for tumorigenesis and an appropriate suicide system such as herpes simplex 1-thymidine kinase transgene $(82,83)$, which will induce cell death under fialuridine treatment, might be required as a safety mechanism to escape potential tumorigenesis from major histocompatibility complex class II-deficient ECFCs. Although these barriers to clinical translation are not trivial, we believe that use of ECFCs for revascularizing damaged tissue will be ready in the near future for human clinical trials.

Disclosure: The authors declare no conflict of interest.

\section{REFERENCES}

1. Tagin MA, Woolcott CG, Vincer MJ, et al. Hypothermia for neonatal hypoxic ischemic encephalopathy: an updated systematic review and meta-analysis. Arch Pediatr Adolesc Med 2012;166:558-66.

2. Manley BJ, Owen LS, Hooper SB, et al. Towards evidence-based resuscitation of the newborn infant. Lancet 2017;389:1639-48.

3. Owen LS, Manley BJ, Davis PG, et al. The evolution of modern respiratory care for preterm infants. Lancet 2017;389:1649-59.

4. Neu J. Preterm infant nutrition, gut bacteria, and necrotizing enterocolitis. Curr Opin Clin Nutr Metab Care 2015;18:285-8.

5. Hartnett ME. Role of cytokines and treatment algorithms in retinopathy of prematurity. Curr Opin Ophthalmol 2017;28:282-8.

6. Tasev D, Koolwijk P, van Hinsbergh VW. Therapeutic potential of human-derived endothelial colony-forming cells in animal models. Tissue Eng Part B Rev 2016;22:371-82.

7. Medina RJ, Barber CL, Sabatier F, et al. Endothelial progenitors: a consensus statement on nomenclature. Stem Cells Transl Med 2017;6: 1316-20.

8. Basile DP, Yoder MC. Circulating and tissue resident endothelial progenitor cells. J Cell Physiol 2014;229:10-6.

9. Asahara T, Murohara T, Sullivan A, et al. Isolation of putative progenitor endothelial cells for angiogenesis. Science 1997;275:964-7.

10. Estes ML, Mund JA, Ingram DA, et al. Identification of endothelial cells and progenitor cell subsets in human peripheral blood. Curr Protoc Cytom 2010 Chapter 9;52:Unit 933 1-11.

11. Estes ML, Mund JA, Mead LE, et al. Application of polychromatic flow cytometry to identify novel subsets of circulating cells with angiogenic potential. Cytometry A 2010;77:831-9.
12. Asahara T, Kawamoto A, Masuda H. Concise review: circulating endothelial progenitor cells for vascular medicine. Stem Cells 2011;29: $1650-5$.

13. Medina RJ, O'Neill CL, O'Doherty TM, et al. Myeloid angiogenic cells act as alternative M2 macrophages and modulate angiogenesis through interleukin-8. Mol Med 2011;17:1045-55.

14. Purhonen S, Palm J, Rossi D, et al. Bone marrow-derived circulating endothelial precursors do not contribute to vascular endothelium and are not needed for tumor growth. Proc Natl Acad Sci USA 2008;105: $6620-5$.

15. Lin Y, Weisdorf DJ, Solovey A, et al. Origins of circulating endothelial cells and endothelial outgrowth from blood. J Clin Invest 2000;105:71-7.

16. Medina RJ, O'Neill CL, Sweeney M, et al. Molecular analysis of endothelial progenitor cell (EPC) subtypes reveals two distinct cell populations with different identities. BMC Med Genomics 2010;3:18.

17. Ingram DA, Mead LE, Moore DB, et al. Vessel wall-derived endothelial cells rapidly proliferate because they contain a complete hierarchy of endothelial progenitor cells. Blood 2005;105:2783-6.

18. Hubert L, Darbousset R, Panicot-Dubois L, et al. Neutrophils recruit and activate human endothelial colony-forming cells at the site of vessel injury via P-selectin glycoprotein ligand-1 and L-selectin. J Thromb Haemost 2014;12:1170-81.

19. Yoder MC. Is endothelium the origin of endothelial progenitor cells? Arterioscler Thromb Vasc Biol 2010;30:1094-3.

20. Ingram DA, Mead LE, Tanaka H, et al. Identification of a novel hierarchy of endothelial progenitor cells using human peripheral and umbilical cord blood. Blood 2004;104:2752-60.

21. Yoder MC, Mead LE, Prater D, et al. Redefining endothelial progenitor cells via clonal analysis and hematopoietic stem/progenitor cell principals. Blood 2007;109:1801-9.

22. Melero-Martin JM, Khan ZA, Picard A, et al. In vivo vasculogenic potential of human blood-derived endothelial progenitor cells. Blood 2007;109:4761-8.

23. Au P, Daheron LM, Duda DG, et al. Differential in vivo potential of endothelial progenitor cells from human umbilical cord blood and adult peripheral blood to form functional long-lasting vessels. Blood 2008;111: 1302-5.

24. Bompais H, Chagraoui J, Canron X, et al. Human endothelial cells derived from circulating progenitors display specific functional properties compared with mature vessel wall endothelial cells. Blood 2004;103:2577-84.

25. Takahashi K, Yamanaka S. A decade of transcription factor-mediated reprogramming to pluripotency. Nat Rev Mol Cell Biol 2016;17:183-93.

26. Choi KD, Yu J, Smuga-Otto K, et al. Hematopoietic and endothelial differentiation of human induced pluripotent stem cells. Stem Cells 2009;27:559-67.

27. James D, Nam HS, Seandel M, et al. Expansion and maintenance of human embryonic stem cell-derived endothelial cells by TGFbeta inhibition is Id1 dependent. Nat Biotechnol 2010;28:161-6.

28. Goldman O, Feraud O, Boyer-Di Ponio J, et al. A boost of BMP4 accelerates the commitment of human embryonic stem cells to the endothelial lineage. Stem Cells 2009;27:1750-9.

29. Sone M, Itoh H, Yamahara K, et al. Pathway for differentiation of human embryonic stem cells to vascular cell components and their potential for vascular regeneration. Arterioscler Thromb Vasc Biol 2007;27:2127-34.

30. Prasain N, Lee MR, Vemula S, et al. Differentiation of human pluripotent stem cells to cells similar to cord-blood endothelial colony-forming cells. Nat Biotechnol 2014;32:1151-7.

31. Kang KT, Coggins M, Xiao C, et al. Human vasculogenic cells form functional blood vessels and mitigate adverse remodeling after ischemia reperfusion injury in rats. Angiogenesis 2013;16:773-84.

32. Schwarz TM, Leicht SF, Radic T, et al. Vascular incorporation of endothelial colony-forming cells is essential for functional recovery of murine ischemic tissue following cell therapy. Arterioscler Thromb Vasc Biol 2012;32:e13-21.

33. Popernack ML, Gray N, Reuter-Rice K. Moderate-to-severe traumatic brain injury in children: complications and rehabilitation strategies. J Pediatr Health Care 2015;29:e1-7. 


\section{Tissue regeneration using ECFCs $\quad$ Review}

34. Zhang Y, Li Y, Wang S, et al. Transplantation of expanded endothelial colony-forming cells improved outcomes of traumatic brain injury in a mouse model. J Surg Res 2013;185:441-9.

35. Huang XT, Zhang YQ, Li SJ, et al. Intracerebroventricular transplantation of ex vivo expanded endothelial colony-forming cells restores blood-brain barrier integrity and promotes angiogenesis of mice with traumatic brain injury. J Neurotrauma 2013;30:2080-8.

36. Ding J, Zhao Z, Wang C, et al. Bioluminescence imaging of transplanted human endothelial colony-forming cells in an ischemic mouse model. Brain Res 2016;1642:209-18.

37. Moubarik C, Guillet B, Youssef B, et al. Transplanted late outgrowth endothelial progenitor cells as cell therapy product for stroke. Stem Cell Rev 2011;7:208-20.

38. Garrigue P, Hache G, Bennis Y, et al. Erythropoietin pretreatment of transplanted endothelial colony-forming cells enhances recovery in a cerebral ischemia model by increasing their homing ability: a SPECT/ CT study. J Nucl Med 2016;57:1798-804.

39. Baker CD, Ryan SL, Ingram DA, et al. Endothelial colony-forming cells from preterm infants are increased and more susceptible to hyperoxia. Am J Respir Crit Care Med 2009;180:454-61.

40. Fujinaga $\mathrm{H}$, Baker CD, Ryan SL, et al. Hyperoxia disrupts vascular endothelial growth factor-nitric oxide signaling and decreases growth of endothelial colony-forming cells from preterm infants. Am J Physiol Lung Cell Mol Physiol 2009;297:L1160-9.

41. Baker CD, Balasubramaniam V, Mourani PM, et al. Cord blood angiogenic progenitor cells are decreased in bronchopulmonary dysplasia. Eur Respir J 2012;40:1516-22.

42. Borghesi A, Massa M, Campanelli R, et al. Circulating endothelial progenitor cells in preterm infants with bronchopulmonary dysplasia. Am J Respir Crit Care Med 2009;180:540-6.

43. Alphonse RS, Vadivel A, Fung M, et al. Existence, functional impairment, and lung repair potential of endothelial colony-forming cells in oxygeninduced arrested alveolar growth. Circulation 2014;129:2144-57.

44. Alphonse RS, Vadivel A, Zhong S, et al. The isolation and culture of endothelial colony-forming cells from human and rat lungs. Nat Protoc 2015;10:1697-708.

45. Baker CD, Seedorf GJ, Wisniewski BL, et al. Endothelial colony-forming cell conditioned media promote angiogenesis in vitro and prevent pulmonary hypertension in experimental bronchopulmonary dysplasia. Am J Physiol Lung Cell Mol Physiol 2013;305:L73-81.

46. Connor KM, Krah NM, Dennison RJ, et al. Quantification of oxygeninduced retinopathy in the mouse: a model of vessel loss, vessel regrowth and pathological angiogenesis. Nat Protoc 2009;4:1565-73.

47. Medina RJ, O'Neill CL, Humphreys MW, et al. Outgrowth endothelial cells: characterization and their potential for reversing ischemic retinopathy. Invest Ophthalmol Vis Sci 2010;51:5906-13.

48. Sakimoto S, Marchetti V, Aguilar E, et al. CD44 expression in endothelial colony-forming cells regulates neurovascular trophic effect. JCI Insight 2017;2:e89906.

49. Bonventre JV, Yang L. Cellular pathophysiology of ischemic acute kidney injury. J Clin Invest 2011;121:4210-21.

50. Brodsky SV, Yamamoto T, Tada T, et al. Endothelial dysfunction in ischemic acute renal failure: rescue by transplanted endothelial cells. Am J Physiol Renal Physiol 2002;282:F1140-9.

51. Burger D, Vinas JL, Akbari S, et al. Human endothelial colony-forming cells protect against acute kidney injury: role of exosomes. Am J Pathol 2015;185:2309-23.

52. Collett JA, Mehrotra P, Crone A, et al. Endothelial colony forming cells ameliorate endothelial dysfunction via secreted factors following ischemia-reperfusion injury. Am J Physiol Renal Physiol 2016;312: F897-907.

53. Sradnick J, Rong S, Luedemann A, et al. Extrarenal progenitor cells do not contribute to renal endothelial repair. J Am Soc Nephrol 2016;27: $1714-26$.

54. Vinas JL, Burger D, Zimpelmann J, et al. Transfer of microRNA-486-5p from human endothelial colony forming cell-derived exosomes reduces ischemic kidney injury. Kidney Int 2016;90:1238-50.
55. Hanahan D, Weinberg RA. Hallmarks of cancer: the next generation. Cell 2011;144:646-74.

56. Naito $\mathrm{H}$, Wakabayashi $\mathrm{T}$, Kidoya $\mathrm{H}$, et al. Endothelial side population cells contribute to tumor angiogenesis and antiangiogenic drug resistance. Cancer Res 2016;76:3200-10.

57. Naito H, Kidoya H, Sakimoto S, et al. Identification and characterization of a resident vascular stem/progenitor cell population in preexisting blood vessels. EMBO J 2012;31:842-55.

58. Bieback K, Vinci M, Elvers-Hornung S, et al. Recruitment of human cord blood-derived endothelial colony-forming cells to sites of tumor angiogenesis. Cytotherapy 2013;15:726-39.

59. Laurenzana A, Biagioni A, D'Alessio S, et al. Melanoma cell therapy: endothelial progenitor cells as shuttle of the MMP12 uPARdegrading enzyme. Oncotarget 2014;5:3711-27.

60. Margheri G, Zoppi A, Olmi R, et al. Tumor-tropic endothelial colony forming cells (ECFCs) loaded with near-infrared sensitive Au nanoparticles: a "cellular stove" approach to the photoablation of melanoma. Oncotarget 2016;7:39846-60.

61. Kang KT, Lin RZ, Kuppermann D, et al. Endothelial colony forming cells and mesenchymal progenitor cells form blood vessels and increase blood flow in ischemic muscle. Sci Rep 2017;7:770.

62. Liu Y, Teoh SH, Chong MS, et al. Vasculogenic and osteogenesisenhancing potential of human umbilical cord blood endothelial colonyforming cells. Stem Cells 2012;30:1911-24.

63. Lin RZ, Moreno-Luna R, Zhou B, et al. Equal modulation of endothelial cell function by four distinct tissue-specific mesenchymal stem cells. Angiogenesis 2012;15:443-55.

64. Souidi N, Stolk M, Rudeck J, et al. stromal cells act as guardians for endothelial progenitors by reducing their immunogenicity after cotransplantation. Stem Cells 2017;35:1233-45.

65. Shafiee A, Patel J, Wong HY, et al. Priming of endothelial colony-forming cells in a mesenchymal niche improves engraftment and vasculogenic potential by initiating mesenchymal transition orchestrated by NOTCH signaling. FASEB J 2017;31:610-24.

66. Buno KP, Chen $\mathrm{X}$, Weibel JA, et al. In vitro multitissue interface model supports rapid vasculogenesis and mechanistic study of vascularization across tissue compartments. ACS Appl Mater Interfaces 2016;8:21848-60.

67. Melero-Martin JM, De Obaldia ME, Allen P, et al. Host myeloid cells are necessary for creating bioengineered human vascular networks in vivo. Tissue Eng Part A 2010;16:2457-66.

68. Bennis Y, Sarlon-Bartoli G, Guillet B, et al. Priming of late endothelial progenitor cells with erythropoietin before transplantation requires the CD131 receptor subunit and enhances their angiogenic potential. J Thromb Haemost 2012;10:1914-28.

69. Lee JH, Lee SH, Choi SH, et al. The sulfated polysaccharide fucoidan rescues senescence of endothelial colony-forming cells for ischemic repair. Stem Cells 2015;33:1939-51.

70. Sarlon G, Zemani F, David L, et al. Therapeutic effect of fucoidanstimulated endothelial colony-forming cells in peripheral ischemia. J Thromb Haemost 2012;10:38-48.

71. Heo SC, Kwon YW, Jang IH, et al. WKYMVm-induced activation of formyl peptide receptor 2 stimulates ischemic neovasculogenesis by promoting homing of endothelial colony-forming cells. Stem Cells 2014;32:779-90.

72. Stalin J, Harhouri K, Hubert L, et al. Soluble CD146 boosts therapeutic effect of endothelial progenitors through proteolytic processing of short CD146 isoform. Cardiovasc Res 2016;111:240-51.

73. Green LA, Njoku V, Mund J, et al. Endogenous transmembrane TNFalpha protects against premature senescence in endothelial colony forming cells. Circ Res 2016;118:1512-24.

74. Kim H, Prasain N, Vemula S, et al. Human platelet lysate improves human cord blood derived ECFC survival and vasculogenesis in three dimensional (3D) collagen matrices. Microvasc Res 2015;101:72-81.

75. Allen P, Kang KT, Bischoff J. Rapid onset of perfused blood vessels after implantation of ECFCs and MPCs in collagen, PuraMatrix and fibrin provisional matrices. J Tissue Eng Regen Med 2015;9:632-6. 
76. Critser PJ, Kreger ST, Voytik-Harbin SL, et al. Collagen matrix physical properties modulate endothelial colony forming cell-derived vessels in vivo. Microvasc Res 2010;80:23-30.

77. Blue EK, DiGiuseppe R, Derr-Yellin E, et al. Gestational diabetes induces alterations in the function of neonatal endothelial colony-forming cells. Pediatr Res 2014;75:266-72.

78. Jarajapu YP, Hazra S, Segal M, et al. Vasoreparative dysfunction of CD34 + cells in diabetic individuals involves hypoxic desensitization and impaired autocrine/paracrine mechanisms. PLoS ONE 2014;9:e93965.

79. Nakatsuji N, Nakajima F, Tokunaga K. HLA-haplotype banking and iPS cells. Nat Biotechnol 2008;26:739-40.
80. Zimmermann A, Preynat-Seauve O, Tiercy JM, et al. Haplotype-based banking of human pluripotent stem cells for transplantation: potential and limitations. Stem Cells Dev 2012;21:2364-73.

81. Abrahimi P, Qin L, Chang WG, et al. Blocking MHC class II on human endothelium mitigates acute rejection. JCI Insight 2016;1: e85293.

82. Nanda D, de Jong M, Vogels R, et al. Imaging expression of adenoviral HSV1-tk suicide gene transfer using the nucleoside analogue FIRU. Eur J Nucl Med Mol Imaging 2002;29:939-47.

83. Chen YT, Bradley A. A new positive/negative selectable marker, puDeltatk, for use in embryonic stem cells. Genesis 2000;28:31-5. 